Thorax (1957), 12, 177.

\title{
TRACHEAL RECONSTRUCTION WITHOUT THE USE OF GRAFTS*
}

\author{
BY
}

\author{
R. S. BARCLAY, N. MCSWAN, AND T. M. WELSH \\ From the Thoracic Surgical Unit, Ruchill Hospital, Glasgow
}

Bronchial anastomosis is now a practicable procedure. It was first successfully performed in dogs and reported by Jackson, Lafkin, Tuttle, and Hampton (1949), and in that same year Tuttle described the first successful anastomosis in the human subject after accidental division of a major bronchus. Later reports followed (Mathey and Oustrieres, 1951), and the healing properties of the bronchial tree, including the trachea, were established.

In resection of the trachea, the ideal repair would seem to be end-to-end anastomosis of the severed ends. Because the lower end of the trachea is fixed by the passage of the left main bronchus under the aortic arch, and the elasticity of the bronchial tree is limited, only a small segment can be completely excised and the repair effected in this way. Rob and Bateman (1949) in studies of six fresh human cadavers estimated that a $2-\mathrm{cm}$. segment is the largest that can be excised from the human trachea and the ends united without undue tension on the suture line. This does not permit radical resection of a tracheal tumour. Consequently most procedures on reconstruction of the trachea concern the use of grafts.

We now report a case of cylindroma involving the lower end of the trachea, carina, and the origins of the right and left main bronchi. The tumour-bearing area, $5 \mathrm{~cm}$. in length, was excised and the subsequent large defect closed using a new technique of mobilization and bronchial anastomosis without resort to grafts and without sacrifice of lung tissue.

\section{CASE REPORT}

On February 8, 1955, a man aged 24 years was admitted to the Thoracic Surgical Unit, Ruchill Hospital, complaining of dyspnoea and stridor. During the previous 16 months he had suffered eight attacks of "pneumonia." Radiographs of the chest showed only inflammatory change in the lower lobe of the

\footnotetext{
* Based on a paper read to the Society of Thoracic Surgeons in November, 1956 .
}

right lung. Bronchoscopy revealed a lobulated tumour extending round the anterior, left, and posterior aspects of the tracheal wall just above the carina, narrowing the lumen to about a quarter of its normal size. A simple tumour was assumed and no biopsy was taken, as immediate operation was deemed advisable. On February 9 through a right posterolateral thoracotomy incision, with the patient in the face-down position, the lower end of the trachea was opened vertically and the tumour removed as completely as possible. Recovery was uneventful. Histologically the tumour proved to be a cylindroma. Repeated subsequent bronchoscopies showed progressive narrowing of the bronchial lumen due to annular extension of the tumour. Bronchial biopsy confirmed recurrence of the cylindroma, but it was only after removal of tissue that it was seen that the tumour had extended to involve the carinal area. Tomography helped to define its limits. On January 26, 1956, a second and more radical operation was undertaken. Using the previous exposure, the trachea was fully mobilized within the thorax (Fig. 1) and the left main bronchus severed from the trachea. Up to this point the anaesthetic gases had been administered through a cuffed endotracheal tube placed high in the trachea. An uncuffed endotracheal tube was then tightly inserted into the left main bronchus via the thoracotomy wound and anaesthesia maintained, using only the left lung (Fig. 2). The trachea was opened posteriorly to expose the tumour and to assess its extent. The trachea was divided above the growth and the right main bronchus divided below it. The tumour-bearing area was thus resected leaving a threeway gap in continuity of $5 \mathrm{~cm}$. By dividing the pulmonary ligament, the right lung was easily elevated until the right main bronchus could be anastomosed to the cut end of the trachea using interrupted silk sutures. The tube was then withdrawn from the left main bronchus and anaesthesia resumed by the normal route. There remained the problem of joining the left main bronchus to the most accessible area of the partially reconstructed bronchial tree. This proved to be the right intermediate bronchus. It was appreciated that an opening in the intermediate bronchus would result in loss of anaesthetic gases and oxygen and that the necessity for inflating the right lung would limit the access to the operation field. Both of these difficulties were overcome by intermittent ventilation of the lung while the opening 


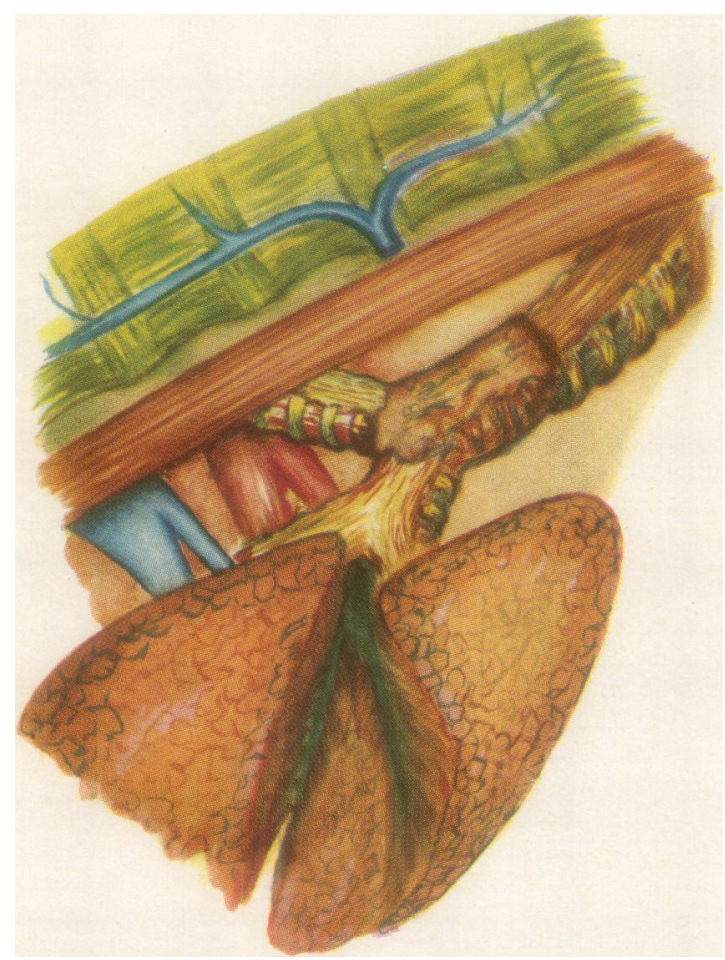

FIG. 1.-The exposure in the face-down position using a right thoracotomy. The extent of mobilization of the trachea and both main bronchi is depicted, also the extent of the tumour.
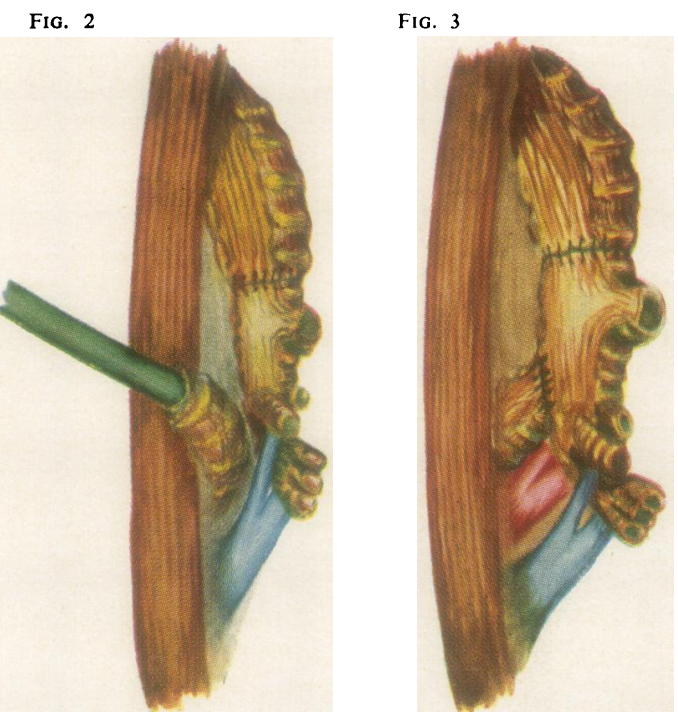

FIG. 2.-Intubation of left main bronchus via the thoracotomy wound. The right bronchial tree and blood vessels are displaced in a cephalad direction to allow anastomosis of the trachea and right main bronchus.

FIG. 3.-Reconstruction completed by anastomosis of the left main bronchus to the side of the right intermediate bronchus.

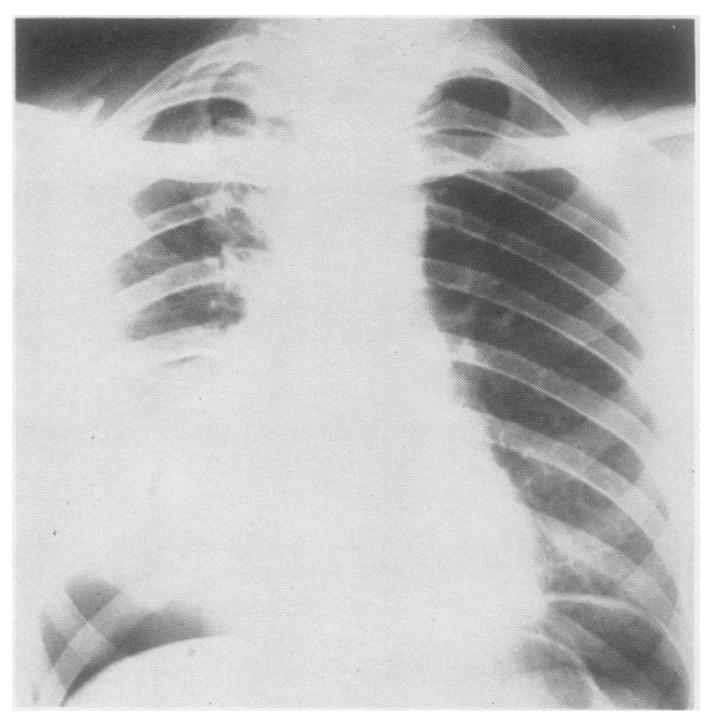

FIG. 4.-Post-operative radiograph of the chest showing pneumoperitoneum.

in the anastomosis area was controlled by digit pressure.

The intermediate bronchus was incised along the medial junction of its membranous and cartilaginoux portions, and after the left main bronchus had been retrimmed an end-to-side anastomosis was made. Continuity had been restored (Fig. 3). The chest was closed and continuous suction maintained through a 3 drainage tube for four days. To ease any strain on the suture line a pneumoperitoneum was induced on the sixth post-operative day and maintained for six weeks (Fig. 4).

Recovery was uneventful and the patient has remained well to date. There is no evidence of $\underset{\dot{\sigma}}{x}$ recurrence. A post-operative bronchogram shows the reconstituted bronchial tree (Fig. 5) and a recent chest film shows normal lung fields (Fig. 6).

\section{Discussion}

In the surgery of tracheal defects repair has been effected by many ingenious operations $\mathcal{N}$ usually involving the use of grafts (Gebauer, N 1950a and b, 1951 ; Abbott, 1950; Abbott, N Van Fleit, and Roberto, 1955; Belsey, 1946, 1950). Ehrlich, Meyer, Taylor, Hass, and Miller (1952), operating on dogs, transplanted $\stackrel{0}{=}$ the right main bronchus into the trachea $4-6 \mathrm{~cm}$. above the carina, and three weeks later performed left pneumonectomy and excised the carina. The operation was successful as a two-stage procedure $\mathbb{D}$ but not when performed in one stage. Grindlay, Clagett, and Moersch (1949), again in a dog, re- 


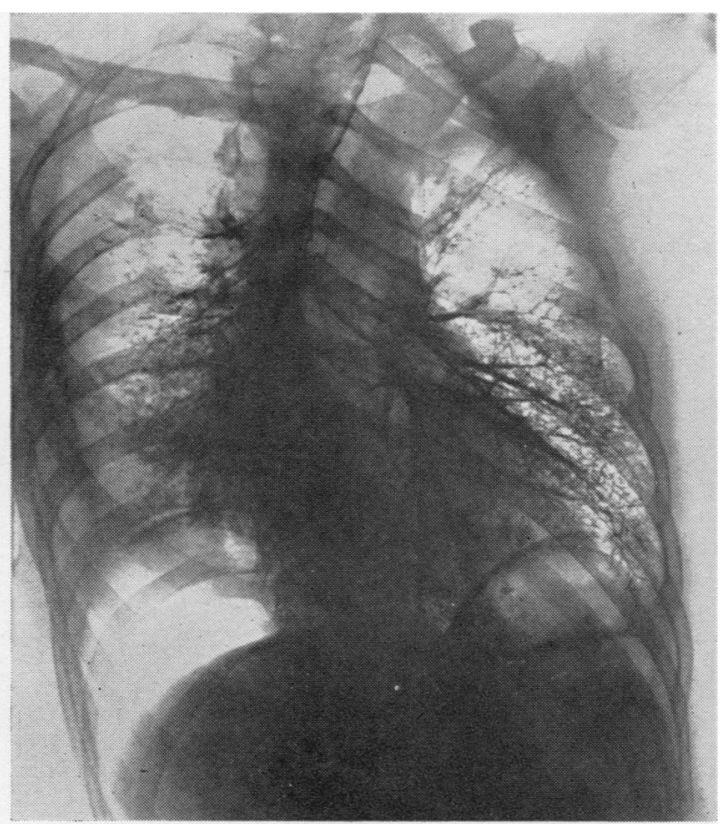

FIG. 5. -Post-operative bronchogram showing reconstituted bronchial tree.

ported resection of the right lung together with a segment of trachea and left main bronchus. The operation was completed by anastomosing the left main bronchus to the trachea. But once more the price of success was sacrifice of a lung. Discussing a paper of Gebauer (1950), Juvenelle (1950), working in the experimental laboratory on dogs, showed that it was possible to excise the carinal area together with segments of the trachea and main bronchi and restore continuity without recourse to grafts or sacrifice of lung tissue. He joined one main bronchus to the trachea by endto-end anastomosis and subsequently implanted the other main bronchus laterally into the trachea above this.

Before the operation we carried out experiments on fresh human cadavers to determine the longest tracheal segment which could be resected and repair effected by direct anastomosis without undue tension on the suture line. Without mobilization of the lower end of the trachea, we confirmed that only $2 \mathrm{~cm}$. could be excised and end-to-end anastomosis achieved. Using the new technique of mobilizing the lower end of the trachea, it was possible to resect $6 \mathrm{~cm}$. of the trachea, including if required the carina and part of the right main bronchus, and reconstitute the bronchial tree as described. Where the tracheal resection did not involve the carina, a third suture line was required to close the opening where the left main bronchus had been severed at the lower end of the trachea.

No reference could be found to pneumoperitoneum as a means of relieving tension on the suture line of a tracheal anastomosis, though its use here seems logical. In the present case, induction of the pneumoperitoneum was delayed until the sixth post-operative day, as it was only then that its possibilities were realized.

Anaesthesia in the type of operation described obviously presents many difficulties, but our experience indicates that they are by no means insuperable.

\section{SUMMARY}

The literature on tracheal reconstruction is reviewed. Hitherto, end-to-end anastomosis has been considered impossible after resection of a tracheal segment longer than $2 \mathrm{~cm}$., and various materials have been used as grafts in such cases.

A case of cylindroma of the trachea, involving the carina and the origins of the right and left main bronchi, is reported, and a new technique is described whereby this carinal tumour was resected and continuity of the bronchial tree restored without recourse to grafts although the gap was $5 \mathrm{~cm}$. in length.

\section{ADDENDUM}

Within recent weeks a second case of tracheal reconstruction has been successfully undertaken in a woman, aged 55 years, for tracheal carcinoma

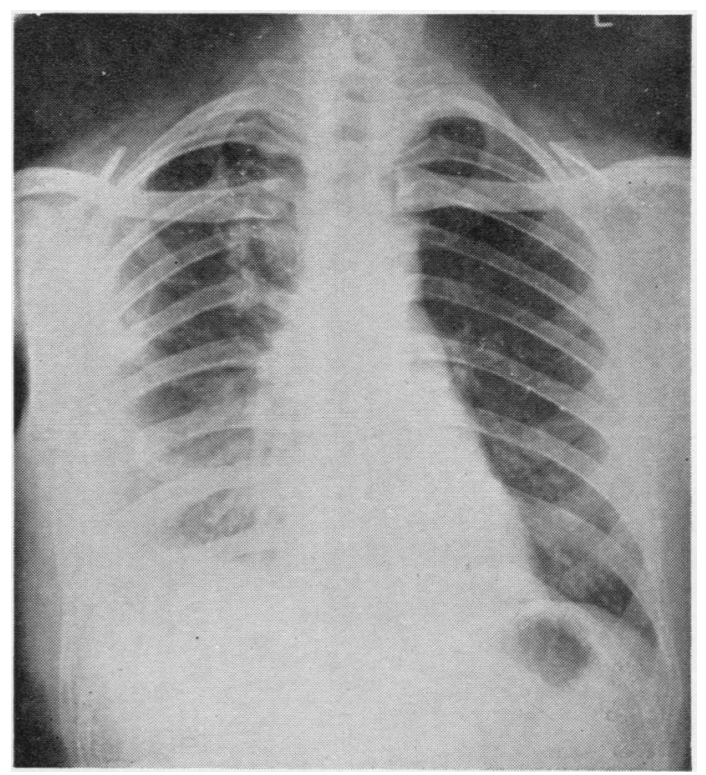

FIG. 6.-Radiograph of the chest one year after operation. 
involving the carina using an identical surgical technique. Convalescence has so far been smooth.

We wish to acknowledge the aid of our anaesthetist colleagues, Drs. Malcolm Shaw and Kenneth Grigor, in the operation on Case 2.

We are indebted to the Endowment Research Fund of the Board of Management of Ruchill Hospital for a grant towards the cost of the colour illustrations.

\section{REFERENCES}

Abbott, O. A. (1950). J. thorac. Surg., 19, 906. -_ Van Fleit, W. E., and Roberto, A. E. (1955). Ibid., 29, 217.
Belsey, R. (1946). Thorax, 1, 39.

- (1950). Brit. J. Surg., 38, 200. Ehrlich, R. W., Meyer, R. P., Taylor, C. B., Hass, G. M., and Mille
E. M. (1952). Surg. Gynec. Obstet., 94, 570.

Gebauer, P. W. (1950a). J. thorac. Surg., 19, 604. - (1950b). Ibid., 20, 628.

- (1951). Ibid., 22, 568.

Grindlay, J. H., Clagett, O. T., and Moersch, H. J. (1949). Pro@ Mayo Clin., 24, 555.

Jackson, T. L., Lafkin. P., Tuttle, W., and Hampton, F. (1949). thorac. Surg., 18, 630.

Juvenelle, A. (1950). Ibid., $20,647$.

Mathey, J., and Oustrieres, G. (1951). Thorax, 6, 71.

Rob, C. G., and Bateman, G. H. (1949). Brit.J. Surg., 37, 202.

Tuttle, W. (1949). J. thorac. Surg., 18, 641. 